\title{
A Global Perspective on Applied Mathematics \& Numerical Analysis
}

\author{
Aisan Khojasteh ${ }^{1}$, Mahmoud Paripour ${ }^{2}$ \\ ${ }^{1}$ M. A. Applied Mathematics, Science and Research Branch, Islamic Azad University, Broujerd, Iran \\ ${ }^{2}$ Assistant Professor of applied Mathematics, Hamedan University of Technology, Hamedan, Iran
}

\section{Email address:}

aisan.khojasteh@yahoo.com (A. khojasteh),Mahmoud_paripour@yahoo.com (M. Paripour)

\section{To cite this article:}

Aisan Khojasteh, Mahmoud Paripour. A Global Perspective on Applied Mathematics \& Numerical Analysis. American Journal of Applied Mathematics. Vol. 2, No. 2, 2014, pp. 69-73. doi: 10.11648/j.ajam.20140202.15

\begin{abstract}
Mathematics has a great variety of applications in the physical sciences. This simple, undeniable fact, however, gives rise to an interesting philosophical problem: why should physical scientists find that they are unable to even state their theories without the resources of abstract mathematical theories? Moreover, the formulation of physical theories in the language of mathematics often leads to new physical predictions which were quite unexpected on purely physical grounds. It is thought by some that the puzzles the applications of mathematics present are artefacts of out-dated philosophical theories about the nature of mathematics. In this paper I examine numerical analysis what precisely it is and why it is important. I begin by presenting a selective conceptual reconstruction of one suggestive line in its historical development. Then expand my focus to a general account of what numerical analysis consists today.
\end{abstract}

Keywords: Applied Mathematics, History, Mathematics, Numerical Analysis, Physical Theories

\section{Introduction}

Numerical analysis studies the means of obtaining numerical results for mathematical expressions: Numerical methods are used ingeniously and indirectly to arrive at the actual numbers implied by a formal solution. While contemporary numerical analysis is inextricably wed to the computer, numerical analysis has its origins in the early 16th century's development of tables of logarithms (Goldstine) which saw mathematicians working to develop the means of generating these tables in increasingly clever and indirect ways in order to avoid cumbersome hand calculations. Logarithms were being used as tools to perform what would have been prohibitively difficult calculations especially in navigation and calculating interest. While it is Napier and Burgi who typically get the lion share of credit for developing logarithms, it was Henry Briggs (1556-1630) who in extending their work, discovered numerical techniques that persist today. Practically any use of mathematics beyond counting involves numerical analysis. This mathematics is often hidden because it is embedded in the calculators, processors, and other computing machinery used to generate the numerical solutions. 1 Although the application of numerical analysis is rarely seen, it is essential to prediction, confirmation, and even explanation in science, as well as in any other setting in which the mathematics involved goes beyond arithmetic including a calcula-tor and its square root function, the calculation of which bear not the slightest resemblance to the hand algorithm taught in grade school.

Numerical analysis involves using mathematical techniques to generate numerical solutions to mathematical expressions. If, for example, you used math to determine that you need to build a wall that is $\sqrt{2}$ meters long, you probably used trigonometry and/or calculus. If you go on to actually find the value of $\sqrt{2}$ to nine decimal places, and then you used numerical analysis. The modern theory of numerical analysis includes developing numerical methods, but it also seeks to ground the techniques in theory to establish why they work, how accurately, how generally, and how robustly. It works to develop the means of generating numerical solutions not just too simple expressions like 2 but also to problems on the cutting edge of science and engineering

\section{Historical Background}

Numerical algorithms are almost as old as human civilization. The Rhind Papyrus ( 1650 BC) of ancient 
Egypt describes a root finding method for solving a simple equation; see [1]. Archimedes of Syracuse (287-212 BC) created much new mathematics, including the "method of exhaustion" for calculating lengths, areas, and volumes of geometric figures; see [2]. When used as a method to find approximations, it is in much the spirit of modern numerical integration; and it was an important precursor to the development of the calculus by Isaac Newton and Gottfried Leibnitz. A major impetus to developing numerical procedures was the invention of the calculus by Newton and Leibnitz, as this led to accurate mathematical models for physical reality, first in the physical sciences and eventually in the other sciences, engineering, medicine, and business. These mathematical models cannot usually be solved explicitly, and numerical methods to obtain approximate solutions are needed. Another important aspect of the development of numerical methods was the creation of logarithms by Napier (1614) and others, giving a much simpler manner of carrying out the arithmetic operations of multiplication, division, and exponentiation. Newton created a number of numerical methods for solving a variety of problems, and his name is attached today to generalizations of his original ideas. Of special note is his work on root finding and polynomial interpolation. Following Newton, many of the giants of mathematics of the 18th and 19th centuries made major contributions to the numerical solution of mathematical problems. Foremost among these are Leonhard Euler (1707-1783), Joseph-Louis Lagrange (1736-1813), and Karl Friedrich Gauss (1777-1855). Up to the late 1800's, it appears that most mathematicians were quite.

\section{Common Perspectives in Numerical Analysis}

Numerical analysis is concerned with all aspects of the numerical solution of a problem, from the theoretical development and understanding of numerical methods to their practical implementation as reliable and efficient computer programs. Most numerical analysts specialize in small sub-areas, but they share some common concerns, perspectives, and mathematical methods of analysis. These include the following.

1. When presented with a problem that cannot be solved directly, then replace it with a "nearby problem" which can be solved more easily. Examples are the use of interpolation in developing numerical integration methods and root finding methods; [3].

2. There is widespread use of the language and results of linear algebra, real analysis, and functional analysis (with its simplifying notation of norms, vector spaces, and operators). [4].

3. There is a fundamental concern with error, its size, and its analytic form. When approximating a problem, as above in item 1, it is prudent to understand the nature of the error in the computed solution. Moreover, understanding the form of the error allows creation of extrapolation processes to improve the convergence behavior of the numerical method.

\section{Effects of Computer Hardware}

First and foremost, the computer arithmetic must be understood. Historically, computer arithmetic varied greatly between different computer manufacturers, and this was a source of many problems when attempting to write software which could be easily ported between different computers. This has been lessoned significantly with the development of the IEEE (Institute for Electrical and Electronic Engineering) standard for computer floating-point arithmetic. All small computers have adopted this standard, and larger computer manufacturers have done so as well. For a discussion of the standard and of computer floating-point arithmetic in general see [5]. For large scale problems, especially in numerical linear algebra, it is important to know how the elements of an array $A$ or a vector $\mathbf{x}$ are stored in memory. Knowing this can lead to much faster transfer of numbers from the memory into the arithmetic registers of the computer, thus leading to faster programs. A somewhat related topic is that of pipelining. This is a widely used technique whereby the execution of computer operations are overlapped, leading to faster execution. Machines with the same basic clock speed can have very different program execution times due to differences in pipelining and differences in the way memory is accessed. Most present-day computers are sequential in their operation, but parallel computers are being used ever more widely. Some parallel computers have independent processors that all access the same computer memory (shared memory parallel computers), whereas other parallel computers have separate memory for each processor (distributed memory parallel computers). Another form of parallelism is the use of pipelining of vector arithmetic operations. Some parallel machines are a combination of some or all of these patterns of memory storage and pipelining. With all parallel machines, the form of a numerical algorithm must be changed in order to make best use of the parallelism for examples of this in numerical linear algebra [6].

\section{Nonlinear Differential and Integral Equations}

Within recent years interest in nonlinear equations has grown enormously. They are extremely important as basic equations in many areas of mathematical physics, and they have received renewed attention because of progress in their solution by machines. This volume undertakes a definition of the field, indicating advances that have been made up through 1960. The author's position is that while the advent of machines has resulted in much new knowledge, one should not disregard analytical methods, since the solution of nonlinear equations possesses singularities which only the analytical method (as based upon the work of Poincare, 
Liapounoff, Pain eve and Goursatl can discover.

After a general survey of the problem presented by nonlinear equations, the author discusses the differential equation of the first order, following this by chapters on the Riccati equation (as a bridge between linear and nonlinear equations) and existence theorems, with special reference to Cauchy's method. Second order equations are introduced via Volterra's problem and the problem of pursuit, and succeeding chapters cover elliptic integrals and functions and theta functions; differential equations of the second order; and second order differential equations of the polynomial class, with special reference to Painleve transcendent. The technique of continuous analytical continuation is shown, while phenomena of the phase plane are studied as an introduction to nonlinear mechanics. Nonlinear 111echanics is then discussed, with various classical equations like Van der Pol's equations, Emden's equation, and the Duffing problem. The remaining chapters are concerned with nonlinear integral equations, problems from the calculus of variations, and numerical integration of nonlinear equations. Throughout the book the results of distinguished analysis of the past and modern machine computations are both taken into account. Despite the thoroughness of its coverage this is a very fine introduction to this important area of mathematics, and it can easily be followed by the mathematically sophisticated reader who knows very little about nonlinear equations for example:

$$
\begin{gathered}
x(t)=\int_{a}^{b} k(t, s) x^{(n)} d s \\
x^{(i)}(0)=d_{i}, 0 \leq i \leq n
\end{gathered}
$$

\section{Method for Ordinary Differential Equations}

This part will introduce the reader to the terminology and notation of differential equations. Students will also be reminded of some of the elementary solution methods they are assumed to have encountered in an undergraduate course on the subject. At the conclusion of this review one should have an idea of what it means to 'solve' a differential equation and some confidence that they could construct a solution to some simple and special types of differential equations for example:

$$
\begin{gathered}
x^{(n)}(t)=y(t)+\int_{a}^{s} k(t, s) x(s) d s \\
x^{(i)}(0)=d_{i}, \quad 0 \leq i \leq n
\end{gathered}
$$

At the core of each of $K(t, s),(3-1),(2-1)$ the equations, the equations say that each and every one square homepage. Obviously, each of the above equations, the kernel and the function $\mathrm{y}(\mathrm{t})$ are known such as:

$$
\begin{array}{ll}
\text { 1) } & \left(K^{*}\right)^{*}=k \\
\text { 2) } & \left(\lambda K^{*}\right)=\lambda k^{*} \\
\text { 3) } & (K h)^{*}=h^{*} k^{*} \\
\text { 4) } & \left(K^{*} x, y\right)^{*}=\left(x, k^{*} y\right)
\end{array}
$$

In case kernel $\mathrm{h}$ and $\mathrm{k}$ is continuous, then:

$$
\begin{aligned}
& (K L)^{*}(s, t)=\overline{(k l)(t, s)} \\
& =\int_{a}^{b} \overline{k(t, u) L(u, s)} d u \\
& =\int_{a}^{b} \overline{k(t, u) L(u, s)} d u \\
& =\int_{a}^{b} L^{*}(s, u) K^{*}(u, t) d u \\
& =L^{*} K^{*}(s, t)
\end{aligned}
$$

Therefore, we consider the following differential general form of an equation integral:

$$
\begin{gathered}
P(t) y^{\prime \prime}(t)+Q(t) y^{\prime}(t)+R(t) y(t)+\lambda \int_{a}^{b} k(t, s) y(s) d s=g(t) \\
C y(r)+D y^{\prime}(r) \\
r=\left(r_{1}, r_{2}, \ldots, r_{m}\right)^{T}, a \leq r_{i} \leq b, 1 \leq i \leq m \\
y(r)=\left(y(r)_{1}, \ldots, y\left(r_{m}\right)^{T}\right. \\
y^{\prime}(r)=\left(y^{\prime}(r)_{1}, \ldots, y^{\prime}\left(r_{m}\right)^{T}\right.
\end{gathered}
$$

\section{The Feather of Numerical Analysis}

Having discussed what animates a small corner of numerical analysis, how it developed, and some of its activities, methods, and concepts, I now consider how the field of numerical analysis unfolds into areas beyond calculating square roots. A standard introduction to numerical analysis will begin with methods of solving equations in one variable that is, finding the $x$ 's, such that $f(\mathrm{x})=\mathrm{o}$ Now as we've seen, the problem of calculating a square root can be transformed into just such a problem, and as you may imagine, so can many others. The methods of solving these equations of one variable get more and more specialized and powerful, and typically the more narrowly one focuses on such a problem, the more efficient the methods get. For example, if one assumes that the function is a polynomial, then the methods available are extremely efficient and stable. Another cottage industry in numerical analysis is the problem of approximating a function for which one knows only a few of its values. 
Typically one does this by determining a "well-behaved" function (like a polynomial) that hits all the known data points and then uses this function to estimate unknown data points. This area is known as polynomial approximation and interpolation. Again it generalizes to more general approximation techniques, including using least square methods, orthogonal polynomials, rational functions, and trigonometric polynomials by way of Fourier transformations. The problems of integration and differentiation are also mainstays of numerical analysis. For any of the real world problems that are solved by calculus (characterized by the operations of differentiation and integration), in order to actually use the solution, numerical results will be needed. Again such methods begin with well-behaved functions of one variable and branch out from there to very complicated functions of multiple variables. In the case of both, the crucial tools are classes of "well-behaved" functions like the polynomials. It can be proven that for any continuous function of interest, a polynomial can be found that is arbitrarily close to the function of interest at every point in a closed interval. So to get a numerical result of a given accuracy to a definite integral or a derivative of a function at a point, one generates an appropriately close polynomial and then treats it as the function of interest to differentiate or integrate. Numerical analysis is further used to prove results concerning error bounds on the difference between the actual and approximate values. Another important area of inquiry concerns how to best choose the original data points by which to generate the approximating function. Adaptive quadrature methods chose the location and density of such node points by analyzing the functional variation and using more nodes in regions of higher variation.

\section{Numerical Analysis as Applied Mathematics}

In the times of Briggs, Newton, and Bolzano (to name a few) the relationship between the formal analytic problems and the numerical were still being worked out. In the wonderful stewpot that was mathematics from the 16th century on, we see the concurrent development of formal notation, logical foundations, analysis, and the topology of the real numbers, which are precisely the theoretical underpinnings of contemporary numerical analysis. In this period numerical concerns were naturally quite visible. I'd like to suggest that some of this persists today. Let me offer the following three rough and ready ways in which numerical analysis functions in scientific application to draw this out. Instrumental Exact solutions are available, e.g., finding the zeros of a complicated polynomial. Essential No exact solution is available (even in principle), e.g., certain differential equations. Explanatory/exploratory the problem is sufficiently complicated that the relationship between the formal mathematical and the numerical is not understood, e.g., general relativity theory. The first role is instrumental in that a closed exact solution is known and the numerical methods are used only to "do the calculations." Imagine an engineer needing to find the zeros of a complicated cubic polynomial — messy work, but work that could be done without numerical analysis. Things get a bit fuzzier when one has a simple first order homogenous differential equation that has a trigonometric solution:

Obtaining the exact solution requires no numerical analysis, but evaluating the trigonometric solution at values of interest does. But more often than not, even in well-understood settings, exact solutions are unavailable. In this case the role of numerical analysis is essential.4 Consider something as straightforward as a second-order differential equation with initial values (a so called "initial value problem"). Such a problem might arise in the modeling of the efficacy of the brakes of a car, taking into account wind resistance and friction. When exact solutions are not available (as they mostly are not), then the only way to

[1]. Confirm that the differential equation models the braking situation and

[2]. Make use of the differential equation to determine a breaking distance is by way of numerical analysis and its highly theoretical grounding in pure mathematics. Nonetheless, the numerical analysis required to perform (1) and (2) is understood to the point that it now covered in "first courses" in numerical analysis. So while essential, these applications of numerical analysis are so routine as to be "invisible." The third explanatory/exploratory way is important here because numerical analysis applications of this kind are clearly not invisible in any sense. These are examples in which the applied mathematical model and its analytic solutions are sufficiently complicated that the relationship between the mathematical model and numerical results are not understood very well at all. Examples like this seem to be present in general relativity, quantum mechanics, and even some classical systems that are chaotic or Brownian. For example, in the study of the dynamics of deterministic systems using differential equations, the numerical methods are well understood. It turns out, however, that most real phenomena have a stochastic or random component; the mathematical tools used to describe such stochastic dynamics are stochastic differential equations. In the period before the rigorous formalization of mathematics, perhaps as far back as Babylonia through the early 17th century (the cut off here is somewhat arbitrary); it is rather anachronistic to interpret any of the work being done as numerical analysis. To be sure much of this early work was foundational for numerical analysis but only because it was foundational for the rigorous formal axiomatic mathematics we have today. Prior to the formal development of analysis, while one can point out mathematical work on numerical techniques, properly speak 348. Anthony F. Peressiniing, no numerical analysis was being done since the means of comparing and classifying convergence rates and error analysis were not 
available. During this period (and ensuing periods leading to modern numerical analysis) the visibility of work on numerical methods was pervasive due to its being explored concurrently with the development of analysis in general. In contrast, contemporary numerical analysis' visibility is limited largely (as we have seen) to its explanatory/exploratory role in scientific application. In the instrumental and even essential roles characterized above, its invisibility is due to the numerical tractability of the theory employing numerical analysis. In such settings, the numerical landscape is charted and it can fade invisibly into the background. In other application settings, ones that are numerically problematic, numerical analysis plays an active and visible role. This difference in the visibility and role of contemporary numerical work in mathematics grew out of two distinct (though perhaps not independent) developments: the modern formalization of analysis and the advent of the computer. Once in place, these two developments allowed the three roles of numerical analysis described above to coalesce into more or less the form we find them in now.

\section{Conclusion}

This paper included analysis of applied mathematics \& analysis numerical. In contrast, contemporary numerical analysis' visibility is limited largely (as we have seen) to its explanatory/exploratory role in scientific application. In the instrumental and even essential roles characterized above, its invisibility is due to the numerical tractability of the theory employing numerical analysis. In such settings, the numerical landscape is charted and it can fade invisibly into the background. In other application settings, ones that are numerically problematic, numerical analysis plays an active and visible role. This difference in the visibility and role of contemporary numerical work in mathematics grew out of two distinct (though perhaps not independent) developments: the modern formalization of analysis and the advent of the computer. Once in place, these two developments allowed the three roles of numerical analysis described above to coalesce into more or less the form we find them in now.

\section{References}

[1] H. Diamond, When do Beurling's generalized integers have density? J. Reine Angew. Math., 295 (1977), 22-39.

[2] C. Edwards, Jr. (1997) The Historical Development of the Calculus, Springer-Verlag.

[3] K. Atkinson (1989) An Introduction to Numerical Analysis, 2nd ed., John Wiley Pub.

[4] P.T. Bateman and H.G. Diamond, Asymptotic distribution of Beurling's generalized prime numbers, J. Studies in Number Theory. Ann, 6 (1969), $152\{212\}$

[5] M. Overton (2001) Numerical Computing with IEEE Floating Point Arithmetic, SIAM Pub.

[6] J. Dongarra, I. Duff, D. Sorensen, and H. van der Vorst (1998) Numerical Linear Algebra for High-Performance Computers, SIAM Pub. 